\title{
Optimized calibration of the adaptive optics system on the LAM Pyramid bench
}

\author{
Charlotte Z. Bond ${ }^{\mathrm{a}}$, Carlos M. Correia ${ }^{\mathrm{a}}$, Jean-François Sauvage ${ }^{\mathrm{a}, \mathrm{b}}$, Kacem El Hadi ${ }^{\mathrm{a}}$, Yannick \\ Abautret $^{\mathrm{a}}$, Benoit Neichel ${ }^{\mathrm{a}}$, and Thierry Fusco ${ }^{\mathrm{a}, \mathrm{b}}$ \\ ${ }^{a}$ Aix Marseille Univ, CNRS, LAM, Laboratoire d`Astrophysique de Marseille, Marseille, France \\ ${ }^{b}$ ONERA - Office National d'Etudes et de Recherches Aérospatiales, B.P.72, F-92322 \\ Châtillon, France
}

\begin{abstract}
The Pyramid wave-front sensor (WFS) is currently the baseline for several future adaptive optics (AO) systems, including the first light systems planned for the era of Extremely Large Telescopes (ELTs). Extensive investigation into the Pyramid WFS aim to prepare for this new generation of AO systems, characterizing its behavior under realistic conditions and developing experimental procedures to optimise performance.

An AO bench at Laboratoire d'Astrophysique de Marseille has been developed to analyze the behavior of the Pyramid and develop the necessary operational and calibration routines to optimize performance. The test bench comprises a Pyramid WFS, an ALPAO $9 \times 9$ deformable mirror $(\mathrm{DM})$, a rotating phase screen to simulate atmospheric turbulence and imaging camera. The Pyramid WFS utilizes the low noise $\mathrm{OCAM}^{2}$ camera to image the four pupils and real time control is realized using the adaptive optics simulation software OOMAO (Object Oriented Matlab Adaptive Optics toolbox). ${ }^{1}$

Here we present the latest experimental results from the Pyramid test bench, including comparison with current Pyramid models and $\mathrm{AO}$ simulations. We focus on the calibration of the $\mathrm{AO}$ system and testing the impact of non-linear effects on the performance of the Pyramid. The results demonstrate good agreement with our current models, in particular with the addition of more realistic elements: non-common path aberrations and the optical quality of the Pyramid prism.
\end{abstract}

Keywords: Pyramid wave-front sensor, adaptive optics, optical gain

\section{INTRODUCTION}

Research into new wave-front sensors has been motivated by the demands of the adaptive optics (AO) systems proposed for the next generation of ground based observatories, the Extremely Large Telescopes (ELTs). In particular the Pyramid wave-front sensor (WFS) has emerged as a prime candidate for the baseline for many of these future systems.

The Pyramid WFS, first proposed by Ragazzoni in, ${ }^{2}$ primarily consists of a 4 sided prism onto which light from a telescope is focused. The four sides of the prism project four images of the telescope pupil, which are realized onto a detector via re-imaging optics. Any local tilt of the wave-front will alter the distribution of the light among the 4 pupils, providing a measurement of the incoming wave-front. The Pyramid WFS is an attractive prospect for current and future $\mathrm{AO}$ systems, with the potential to perform better than the commonly used Shack-Hartmann WFS. In particular the Pyramid can provide more sensitive wave-front measurements within the AO correction band, whilst exhibiting reduced susceptibility to aliasing. ${ }^{3}$ Demonstration on the Large Binocular Telescope $e^{4,5}$ has provided a compelling case for its use on future large telescopes.

In order to fully exploit the potential of the Pyramid WFS, its behavior under realistic operating conditions must be fully understood. This is of paramount importance as we enter into the design phase for the AO systems of the ELTs, such as HARMONI. ${ }^{6}$ With this aim an experimental test bench has been set up at Laboratoire d'Astrophysique de Marseille (LAM). Here we present the experimental bench and summarize the

Contact Charlotte Z. Bond: czbond@hawaii.edu 
latest experimental results. Previous work presented on the LAM bench includes: details of the design of the Pyramid WFS and initial bench assembly $;^{7,8}$ a study on different algorithms for processing the Pyramid signals; ${ }^{9}$ and presentation of the final $\mathrm{AO}$ system and initial closed loop results. ${ }^{10}$

\section{PYRAMID EXPERIMENT AT LABORATOIRE D'ASTROPHYSIQUE DE MARSEILLE}

\subsection{Optical layout}

Figure 1 illustrates the optical layout of the LAM Pyramid WFS experiment. The experiment includes:

- Monochromatic light in the form of a laser with $\lambda=660 \mathrm{~nm}$.

- A rotating phase screen, to simulate the effect of a turbulent atmosphere, providing an effective turbulence of $\frac{d}{r_{0}}=3.2$, where $d$ is the actuator spacing and $r_{0}$ is the Fried parameter.

- AO correction provided by an ALPAO deformable mirror (DM), with 9 actuators across the pupil (67 actuators in total).

- A fast tip-tilt mirror which modulates the beam focused onto the Pyramid to adjust the linear range of the sensor. The modulation is provided at $500 \mathrm{~Hz}$ with amplitudes up to $10 \frac{\lambda}{D}$.

- The Pyramid WFS, incorporating an OCAM ${ }^{2}$ camera to realize the four images of the pupil, with 60 pixels across the pupil. The over-sampling, with respect to the DM, allows for future upgrades with a higher number of actuators across the pupil.

- An imaging camera to provide a measurement of the final PSF, providing a means of assessing the performance of the AO system.

- A Shack-Hartmann WFS with 32 lenlets across the pupil to provide comparative wave-front measurements.

- Flat mirrors for alignment and reference measurements.

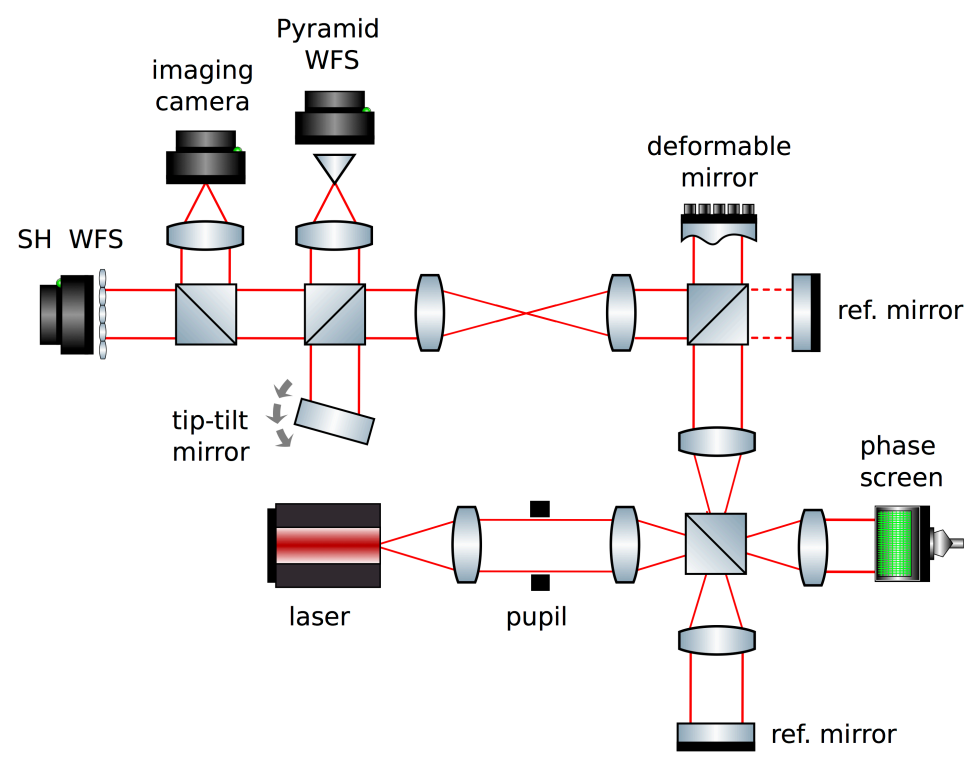

Figure 1. Diagram of the optical layout of the Pyramid experiment at Laboratoire d'Astrophysique de Marseille. Different optical paths are available, in order to test the system in non-turbulent conditions and provide calibration measurements by way of flat reference mirrors. Measurements of the final PSF and the wave-front are made using an imaging camera, Shack-Hartman WFS and the Pyramid WFS. 


\subsection{Controlling the optical bench}

Control of the bench in order to close the loop involves processing the Pyramid signals and sending commands to the deformable mirror. The Pyramid signals are computed as the normalized difference in $x$ and $y$ between the four pupils:

$$
S_{x}[n, m]=\frac{I_{1}[n, m]+I_{3}[n, m]-I_{2}[n, m]-I_{4}[n, m]}{I_{\text {tot. }}} \quad S_{y}[n, m]=\frac{I_{1}[n, m]+I_{2}[n, m]-I_{3}[n, m]-I_{4}[n, m]}{I_{\text {tot. }}}
$$

where $I_{j}$ is the intensity of the equivalent pixel $[n, m]$ in the $j$-th pyramid pupil. $I_{\text {tot. }}$ is the average intensity contained within the valid regions of the 4 pupil images. ${ }^{9}$

The AO system is controlled using cameralink to communicate with the OCAM ${ }^{2}$ camera and the ALPAO Matlab interface to send commands to the DM. The real time control is performed in MatlaB using the AO simulation code OOMAO ${ }^{1}$ allowing for easy comparison between experiment and simulation and ease of testing different algorithms. The code is used to calibrate the system, taking an interaction matrix and then inverting it to provide a command matrix, used to convert the Pyramid signals into DM commands. In closed loop these commands are sent to the deformable mirror to correct for the measured turbulence. The camera is operated at $500 \mathrm{~Hz}$, matched by the modulator speed. The time taken for a complete set of DM commands to be applied to the $\mathrm{DM}$ is $\approx 1 \mathrm{~ms}$, resulting in a top $\mathrm{AO}$ loop speed of $\sim 300 \mathrm{~Hz}$.

\subsection{System characterisation}

To optimize the performance of the AO system, and to accurately predict and compare with models we require detailed knowledge of the AO system. In this section we summarize some of the characterization processes carried out.

\subsubsection{Characterizing the phase screen}

To access the final behavior of the AO loop we need to know the strength of the turbulence we are simulating on the bench. Using the Shack-Hartmann WFS measurements of the phase screen were taken at different rotational intervals. The measurements were decomposed into Zernike polynomials and their variance computed, see figure 2. From these measurements the Fried parameter, $r_{0}$, with respect to the actuator spacing, $d$, was extracted, resulting in a value of $\frac{d}{r_{0}}=3.2$. This is representative of typical values expected for the ELTs (i.e. $\left.d=50 \mathrm{~cm} r_{0}=15.5 \mathrm{~cm}\right)$.

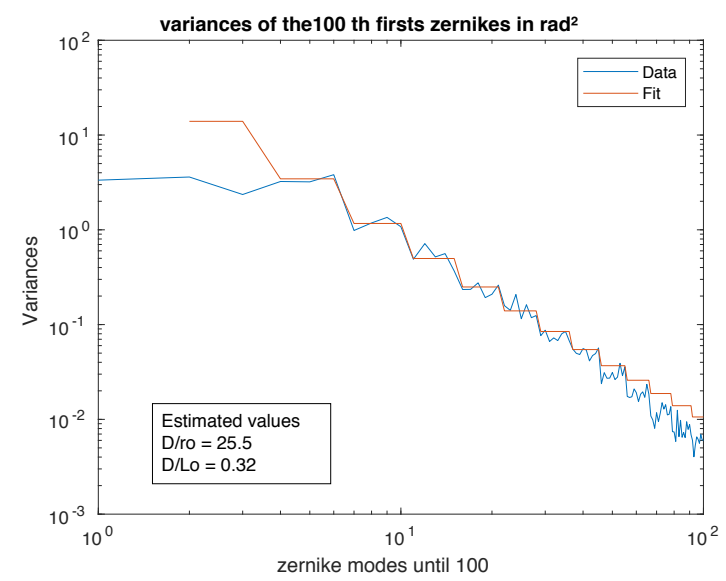

Figure 2. Plot showing the variance of the Zernike modes measured from the phase screen installed on the Pyramid bench. A fitted curve is shown, corresponding to an atmospheric turbulence of $\frac{D}{r_{0}}=25.5$ where $D$ is the pupil diameter. With the current AO system this gives $\frac{d}{r_{0}}=3.2$, where $d$ is the actuator spacing. This work was carried out by Yannick Abautret during an internship at LAM. 


\subsubsection{Modulator calibration}

The behavior of the Pyramid is strongly dependent on the level of modulation. This is often referred to in units of $\frac{\lambda}{D}$. It is crucial that we accurately calibrate the modulation in order to compare the performance with our models. This was carried out by placing a camera at the focal plane of the Pyramid WFS and measuring the amplitude of the modulation, first individually calibrating the $x$ and $y$ axes and finally monitoring the quality of the modulation circle. This work was carried out by Yannick Abautret during an internship at LAM.

\subsubsection{Characterisation of non-common path aberrations}

Non-common path aberrations (NCPA) refer to spatial differences in the optical path between the WFS and imaging camera. To produce the best PSF on the imaging camera the NCPA should be quantified and used to offset the WFS signals to compensate.

On the LAM bench the NCPA was characterized using phase diversity, ${ }^{11,12}$ in order to maximize the Strehl ratio on the imaging camera when the loop is closed. Three images were used, one image in focus, one with positive focus and one with negative focus. The result, using the first 20 Zernike modes, was an estimate of the NCPA with a wave-front error of $21.5 \mathrm{~nm}$ rms. The modal breakdown is summarized in figure 3, showing the majority of the NCPA is astigmatism (mode number 5 ).
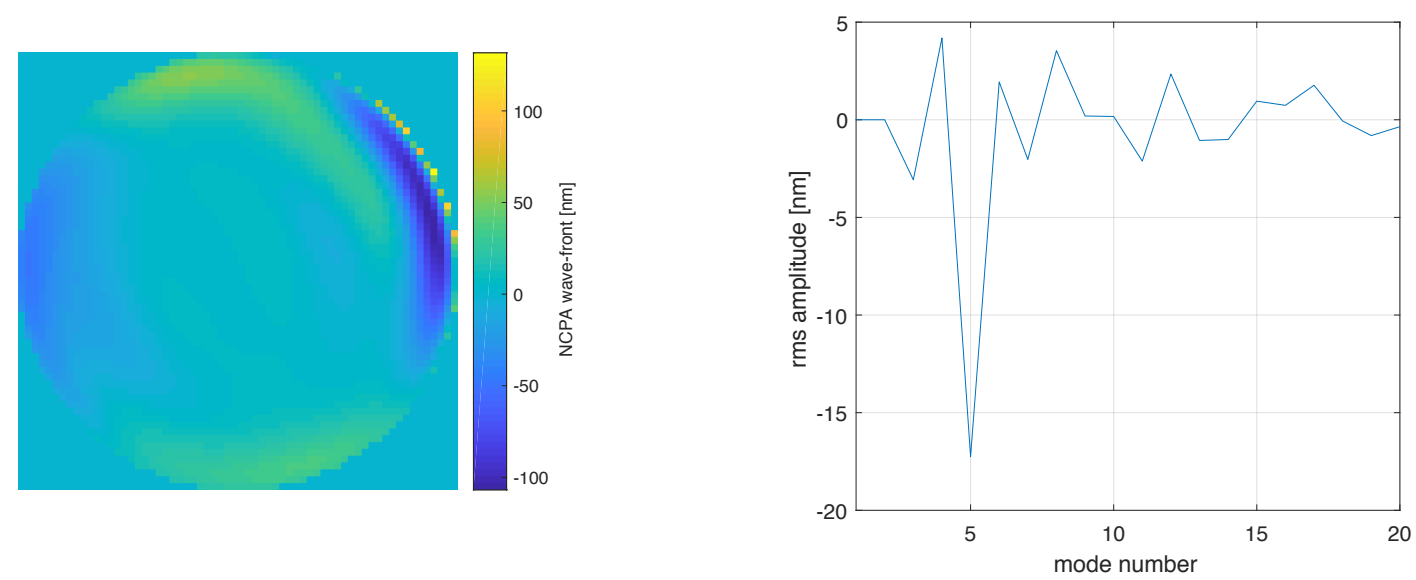

Figure 3. Non-common path aberrations (NCPA) on the LAM bench estimated using phase diversity. Left: Surface map of estimated NCPA, corresponding to an rms wave-front error of $21.5 \mathrm{~nm}$. Right: Zernike coefficients of the first 20 modes estimated in the NCPA (up to radial order $n=5$ ).

\section{INITIAL PYRAMID MEASUREMENTS AND AO CALIBRATION}

\subsection{Measurements with flat wave-fronts}

Once the optical bench was assembled and aligned the first measurements using the Pyramid were taken. Firstly a flat wave-front was sent to the Pyramid WFS, by flattening the deformable mirror. These first measurements are shown in figure 4 . The images shown here show the 4 pupil images for different modulation amplitudes. The pupil configuration has not been specifically optimized for this optical setup and could easily be improved. The crucial aspect is to have enough pixels across the pupil to measure all the modes provided by the DM and to provide sufficient separation of the pupils to avoid interference, both of which are achieved here. Comparing the different modulation amplitudes shown here, we can see that without modulation there is a considerable amount of diffracted light outside the pupils. We also observe an imbalance in the light falling in the different pupils, with more light directed along one diagonal than the other. This is indicative of a 'roof-top' at the tip of the Pyramid prism and causes a drop in sensitivity for spatial modes oriented along the faint diagonal. As the beam is modulated about the Pyramid tip the impact of the roof-top is reduced, resulting in a more uniform distribution of light into the 4 pupils. 

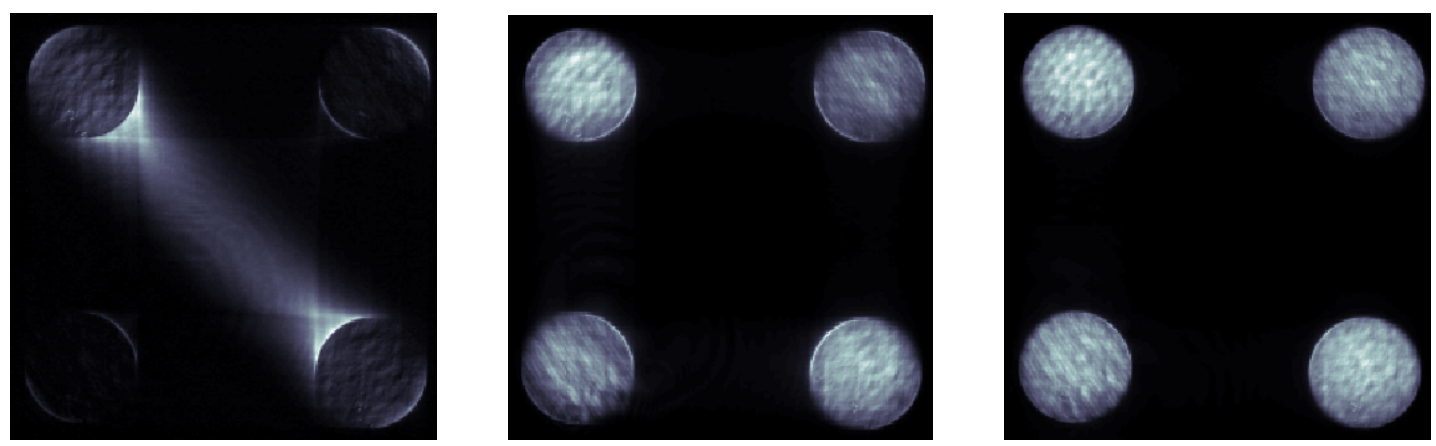

Figure 4. The four pupil images produced by the Pyramid WFS on the LAM bench for a flat wave-front. The 3 different cases shown here correspond to different modulations, from left to right: no modulation; $2 \frac{\lambda}{D}$ modulation; and $5 \frac{\lambda}{D}$ modulation.

These first measurements illustrate the importance of taking a reference WFS measurement corresponding to a flat wave-front. ${ }^{13}$ Without this measurement the AO loop will try to compensate such effects with a non-flat wave-front. Such a reference measurement can also include offsets in order to produce the best possible image on the science camera. We will examine this in more detail in section 4.2 .

\subsection{Calibrating the Pyramid AO system}

To close the loop we first calibrate the system in terms of the relationship between the deformable mirror modes and the Pyramid WFS signals (measuring the interaction matrix). Figure 5 shows the WFS measurements when an interaction matrix is taken using Zernike modes for a modulation of $3 \frac{\lambda}{D}$. In this case each mode corresponds to the same wave-front rms $(10 \mathrm{~nm})$ and the WFS signals demonstrate the variable sensitivity of the Pyramid to different modes (specifically different spatial frequencies). The modulated Pyramid exhibits the response of a slope sensor at low spatial frequencies (below the spatial frequency equivalent to the modulation amplitude). ${ }^{3}$ Above this the response flattens out. This is exhibited here, with some variation over the different modes within one radial order. The consequence of this is, when the interaction matrix is taken with a fixed modal rms the resulting Pyramid signals will vary in strength across the different modes, with the lower orders exhibiting weaker signals with greater susceptibility to measurement noise. For control of the LAM bench we implemented measurement of the interaction matrix using different modal amplitudes to achieve a roughly flat response. The WFS signals for such a measurement are shown in figure 6 .
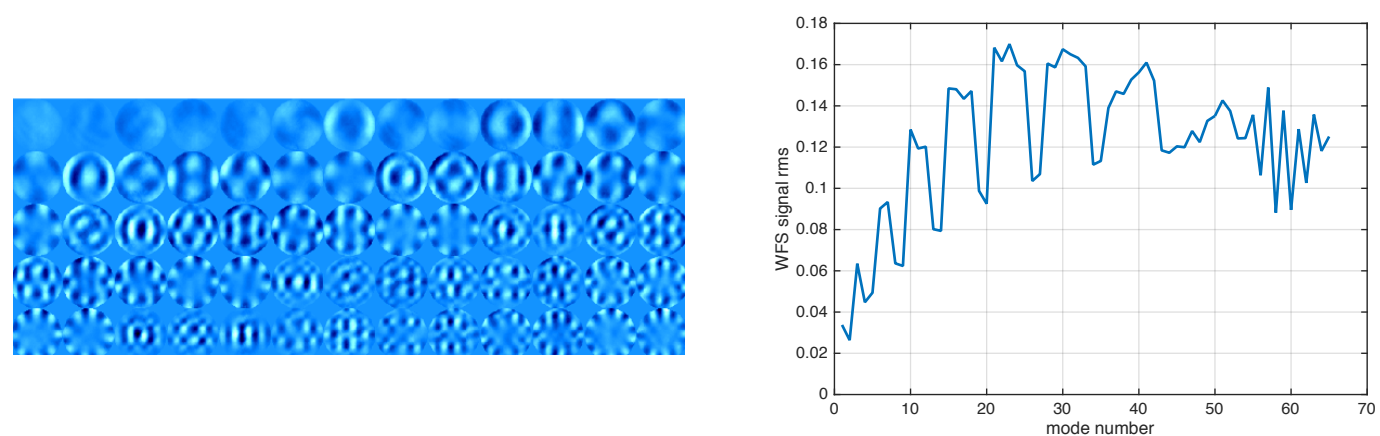

Figure 5. Pyramid WFS signals measured for the first 65 Zernike modes during measurement of an interaction matrix on the LAM bench. Each modes corresponds to $10 \mathrm{~nm}$ rms wave-front error and a modulation of $3 \frac{\lambda}{D}$ was used. Left: WFS signal $S_{x}$ for the first 65 Zernike modes. Right: Average (over $S_{x}$ and $S_{y}$ ) WFS signal rms for the first 65 Zernike modes. 

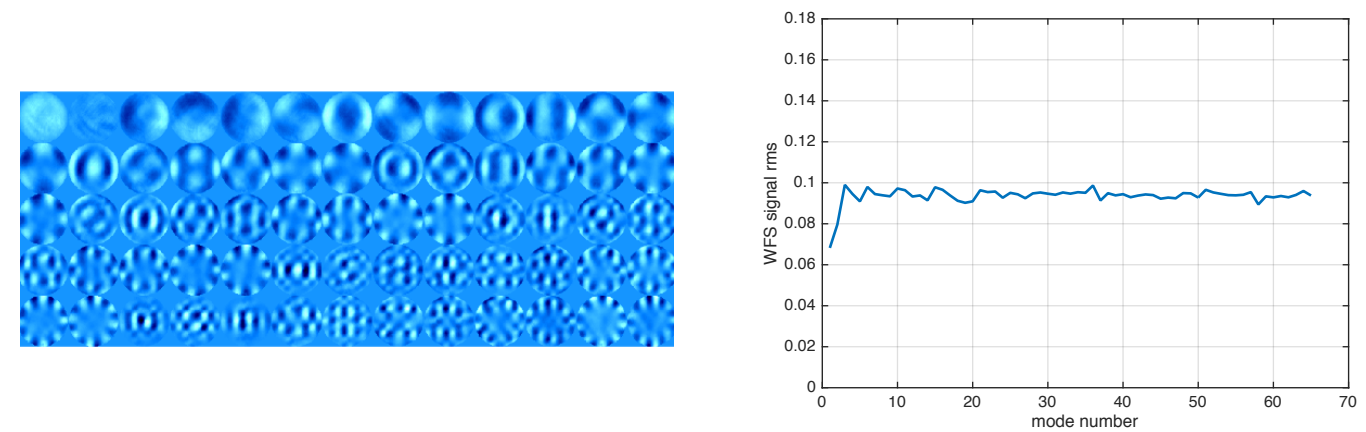

Figure 6. Pyramid WFS signals measured for the first 65 Zernike modes during measurement of an interaction matrix on the LAM bench. The amplitude of each mode is chosen to produce a WFS signal rms of $\sim 0.1$ and a modulation of $3 \frac{\lambda}{D}$ was used. Left: WFS signal $S_{x}$ for the first 65 Zernike modes. Right: Average (over $S_{x}$ and $S_{y}$ ) WFS signal rms for the first 65 Zernike modes.

\section{NON-LINEAR EFFECTS AND OPTICAL GAIN}

\subsection{Linear range}

The Pyramid WFS is often compared favorably with the Shack-Hartmann WFS in terms of the sensitivity of the sensor to the incoming wave-front. Another aspect to consider is the linear response of the sensors. The Pyramid wave-front sensor has a relatively short linear range, particularly when working without modulation. Modulating the Pyramid allows for increasing the linear range of the sensor, at the expense of sensitivity. Ideally the modulation can be tuned to optimize the sensitivity and linearity for given conditions, i.e. for better or worse atmospheric seeing.

Measurements of the response of the Pyramid WFS on the LAM bench were taken by applying a ramp of a given mode to the deformable mirror in open loop. The results for two different modes are shown in figure 7 , using different modulation amplitudes. The low order mode (focus) demonstrates an increase in linear range as the modulation is increased from $1-3 \frac{\lambda}{D}$. The higher order mode $(n=6, m=2)$ requires a larger modulation amplitude to observe a noticeable difference in linear range. The higher the spatial frequency the greater the
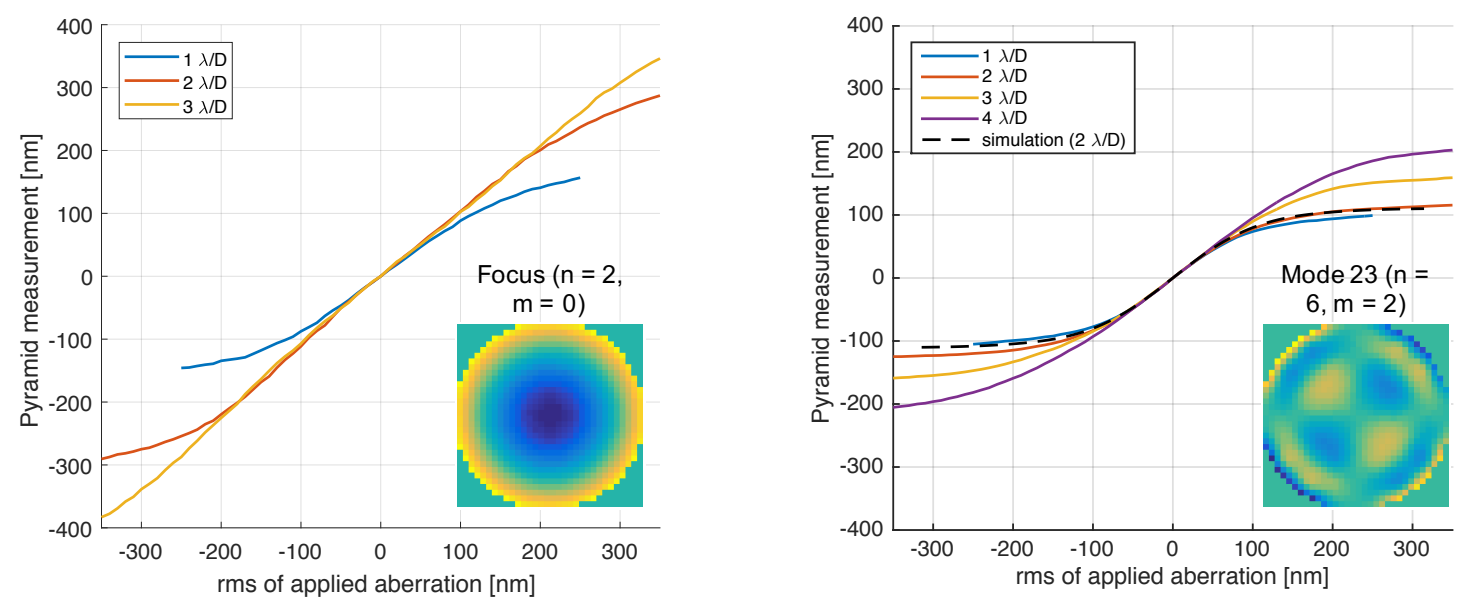

Figure 7. Response of the Pyramid WFS to an applied wave-front, where the amplitude of the applied wave-front is varied. The response is measured for different modulations of the Pyramid and for different spatial modes: focus (left) and a higher order Zernike mode with $n=6$ and $m=2$ (right). In the higher order mode case a simulation result is plotted for comparison. 
modulation amplitude required to affect the Pyramid WFS response. This is related to the point at which the modulation equivalent spatial frequency $\left(\frac{m}{\lambda}\right)$ is greater than or equal to the spatial frequency of the mode.

\subsection{Pyramid operation off-null}

So far we have considered the Pyramid operating around 0, where 0 represents a flat wave-front and corresponds to a diffraction limited PSF focused onto the tip of the Pyramid. Expected operating conditions for the ELTs will degrade the PSF. This can be due to offsets applied to the Pyramid WFS operating point, required to compensate for non-common path aberrations (NCPA) between the WFS and science camera; or to the residual phase errors left after AO correction. The result is a wider PSF which reduces the signal produced by a given wave-front. A sensor calibrated under nominal conditions (a diffraction limited PSF) can be described as having a reduced optical gain, or reduced sensitivity, when operating under realistic conditions.

A reduced optical gain can impact the performance of the Pyramid wave-front sensor as it underestimates the incoming wave-front. When working with an offset applied to the WFS this can result in the offset being incorrectly applied and, for example, an over-correction of any NCPA. This is illustrated in figure 8. Here a focus offset is applied to the WFS during closed loop operation, applying this offset directly to the reconstructed modes. For comparison the open loop Pyramid response is also plotted, along with a linear reference.

When the applied offset is small in amplitude (within the linear range) the system is able to correctly apply the offset in closed loop. However, a larger offset results in an underestimate of the measured wave-front, shown here by the deviation of the open loop response from linear. The consequence in closed loop is an over-application of the offset. The focus measurement saturates around $140 \mathrm{~nm}$, as shown by the maximum of the open loop measurement. If an offset $>140 \mathrm{~nm}$ is attempted the system will always measure the applied offset as $140 \mathrm{~nm}$, causing the control system to continuously apply a larger offset, in an attempt to get the measurement to the set value. The loop at this point becomes unstable.

This result is illustrative of the potential problem when trying to compensate for a large NCPA. Attempts to mitigate this effect (within the stable region) have lead to the proposal of schemes for tracking and correcting the optical gain and correctly applying NCPA correction. ${ }^{14,15}$

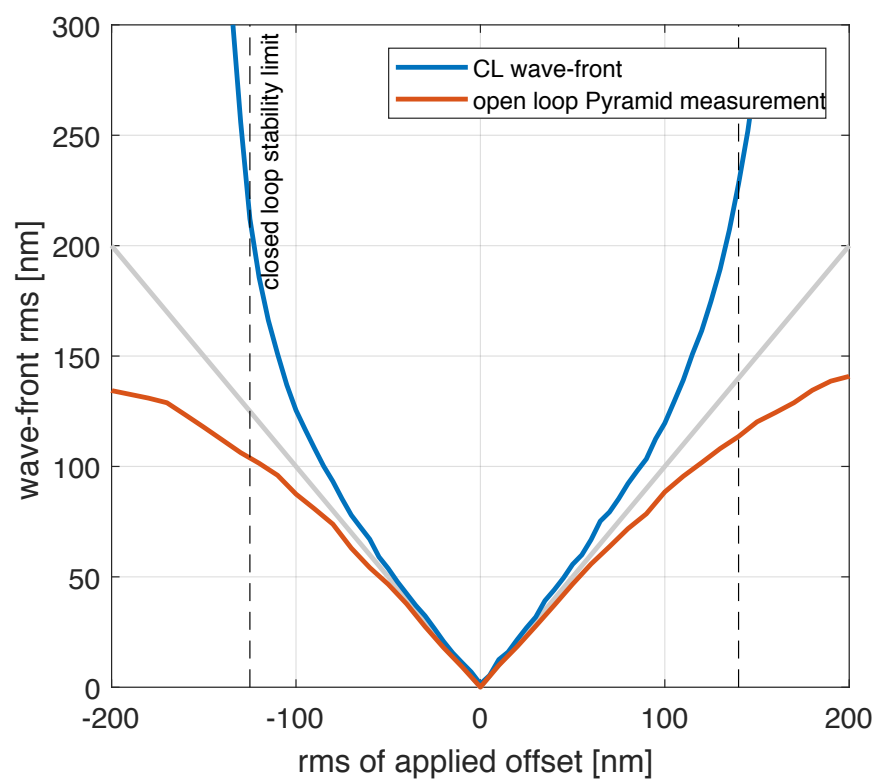

Figure 8. Plot showing the effective closed loop wave-front error introduced when an offset is applied modes reconstructed from Pyramid WFS signals. The example shown here applies a focus offset and shows the rms of the closed loop wave-front vs. the applied offset. Also shown is the open loop response of the Pyramid wave-front sensor and a linear reference (grey line). 

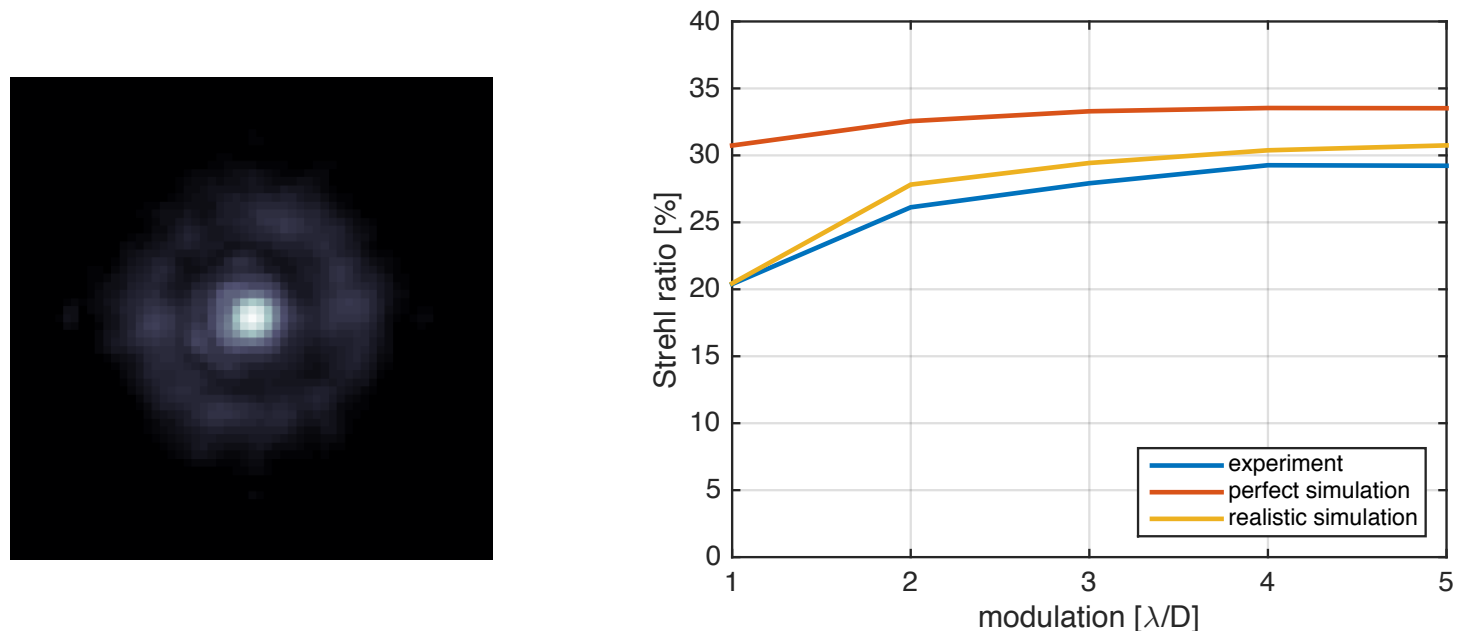

Figure 9. Closed loop results on the LAM Pyramid bench. Left: Long exposure PSF for a modulation of $4 \frac{\lambda}{D}$, corresponding to a Strehl ratio of $\sim 30 \%(\lambda=660 \mathrm{~nm})$. Right: Strehl ratio in closed loop versus modulation. Simulation results are shown for comparison, a 'perfect' model and a realistic simulation.

\section{CLOSING THE LOOP}

Here we present the performance of the LAM Pyramid bench in closed loop, with the phase screen providing dynamic turbulence $\left(\frac{d}{r_{0}}=3.2\right)$ and the loop controlled using Zernike modes. The loop is closed using different modulation amplitudes and assessed by the PSF on the imaging camera, specifically the Strehl ratio.

Figure 9 summarizes the performance in closed loop. The long exposure image demonstrates a Strehl of up to $30 \%$ (with high modulation). With low modulation the performance drops to $\sim 20 \%$. Although a low modulation produces higher sensitivity measurements, the impact of any optical imperfections or non-linear effects is greater, leading to a drop in performance. This can be observed by comparing the experimental results with two simulations. A perfect simulation exhibits a flatter response, with a Strehl ratio between 30 and $35 \%$. The realistic simulation includes:

- The roof-top effect on the tip of the Pyramid

- Non-common path aberrations

- Zernike modes projected onto the DM

Including these effects produces a result close to that observed on the bench, exhibiting a greater variation in Strehl ratio across the range of modulation and specifically sees the larger drop at low modulation. This result provides confidence in both our models of the Pyramid WFS and the experimental bench, as we are able to recreate the experimental results and explain our observations.

\section{CONCLUSION}

Here we have presented the latest experimental results achieved on the Pyramid WFS bench at Laboratoire d'Astrophysique de Marseille. Our system uses a Pyramid WFS to close the loop using a $9 \times 9$ ALPAO DM, a tip-tilt mirror to provide modulation and a phase screen to provide turbulence representative of that expected for ELTs. Real time control is provided by the simulation OOMAO ${ }^{1}$ in MATLAB. The AO system is calibrated, first taking reference signals for a flat wave-front and then measuring an interaction matrix to calibrate the response of the Pyramid WFS to the DM modes. On the LAM bench the amplitude of the modes used in the measurement of the interaction matrix are tuned to provide a flat modal response (in terms of the WFS signals). The loop is closed using a reference signal to compensate for NCPA between the imaging camera and WFS, as estimated 
using phase diversity. A maximum Strehl ratio of $\sim 30 \%$ is achieved at the WFS wavelength $(\lambda=660 \mathrm{~nm})$, with a dip in performance at low modulation due to the impact of non-linear effects and the optical quality of the Pyramid. These results are in good agreement with simulations of the bench, especially when realistic effects such as NCPA are included.

In addition to closing the loop with the Pyramid, we have begun to investigate the non-linear behavior of the Pyramid WFS and in particular the impact when working with an offset applied to the AO loop. We have shown that the small linear range of the Pyramid can result in an underestimate of the incoming wave-front and, in the case of off-null operation, an over application of the offset modes. In order to exploit the full sensitivity of the Pyramid WFS we will need to further investigate these issues and devise methods of compensating for the reduced optical gain experienced under realistic operating conditions. Such work is currently underway.

\section{ACKNOWLEDGMENTS}

The research leading to these results received the support of the A*MIDEX project (no. ANR-11-IDEX-000102) funded by the Investissements d'Avenir French Government program and managed by the French National Research Agency (ANR). This research was co-funded by the People Programme (Marie Curie Actions) of the European Union's Seventh Framework Programme (FP7/2007-2013) under REA grant agreement n. PCOFUNDGA-2013-609102, through the PRESTIGE programme coordinated by Campus France.

\section{REFERENCES}

[1] Conan, R. and Correia, C., "Object-oriented matlab adaptive optics toolbox," in [Proceedings of the SPIE], 9148, 9148 - 17 (2014).

[2] Ragazzoni, R., "Pupil plane wavefront sensing with an oscillating prism," J. of Modern Optics 43, 289-293 (1996).

[3] Vérinaud, C., "On the nature of the measurements provided by a pyramid wave-front sensor," Optics Communications 233, 27-38 (2004).

[4] Esposito, S., Riccardi, A., Quirós-Pacheco, F., Pinna, E., Puglisi, A. T., Xompero, M., Briguglio, R., Busoni, L., Fini, L., Stefanini, P., Brusa, G., Tozzi, A., Ranfagni, P., Pieralli, F., Guerra, J. C., Arcidiacono, C., and Salinari, P., "Laboratory characterization and performance of the high-order adaptive optics system for the large binocular telescope," Applied Optics 49 (2010).

[5] Esposito, S., Riccardi, A., Pinna, E., Puglisi, A. T., Quirós-Pacheco, F., Arcidiacono, C., Xompero, M., Briguglio, R., Busoni, L., Fini, L., Argomedo, J., Gherardi, A., Agapito, G., Brusa, G., Miller, D. L., Ramon, J. C. G., Boutsia, K., and Stefanini, P., "Natural guide star adaptive optics systems at lbt: Flao commissioning and science operations status," in [Proceedings of the SPIE], 8447 (2012).

[6] Thatte, N. A. et al., "Harmoni: the first light integral field spectrograph for the e-elt," in [Proceedings of the SPIE], 9147 (2014).

[7] Hadi, K. E., Fusco, T., and Roux, B. L., "Toward an experimental validation of new ao concepts for future e-elt instrumentation," in [Proceedings of the SPIE], (2012).

[8] Hadi, K. E., Vignaux, M., and Fusco, T., "Development of a pyramid wavefront sensor," in [Adaptive Optics for Extremely Large Telescopes 3 - Conference Proceedings], (2013).

[9] Bond, C. Z., Hadi, K. E., Sauvage, J. F., Correia, C., Fauvarque, O., Rabaud, D., Neichel, B., and Fusco, T., "Experimental implementation of a pyramid wfs: Towards the first scao systems for e-elt," in [Adaptive Optics for Extremely Large Telescopes 4 - Conference Proceedings], (2015).

[10] Bond, C. Z., Hadi, K. E., Sauvage, J.-F., Correia, C., Fauvarque, O., Rabaud, D., Lamb, M., Neichel, B., and Fusco, T., "Experimental study of an optimised pyramid wave-front sensor for extremely large telescopes," in [Proceedings of the SPIE], (2016).

[11] Lamb, M., Andersen, D. R., Véran, J.-P., Correia, C., Herriot, G., Rosensteiner, M., and Fiege, J., "Noncommon path aberration corrections for current and future ao systems," in [Proceedings of the SPIE], 9148 (2014). 
[12] Lamb, M. P., Correia, C., Sauvage, J.-F., Véran, J.-P., Andersen, D. R., Vigan, A., Wizinowich, P. L., van Dam, M. A., Mugnier, L., and Bond, C., "Quantifying telescope phase discontinuities external to adaptive optics systems by use of phase diversity and focal plane sharpening," J. of Astronomical Telescopes, Instruments and Systems 3 (2017).

[13] Fauvarque, O. PhD thesis (2017).

[14] Esposito, S., Pinna, E., Puglisi, A., Agapito, G., Véran, J.-P., and Herriot, G., "Non common path aberration correction with non linear wfss," in [Adaptive Optics for Extremely Large Telescopes 4 - Conference Proceedings], (2015).

[15] Bond, C. Z. et al., "Optimising the performance of a pyramid wfs: tracking the optical gain," (in preparation). 